\title{
Wie können Abrufübungen (nicht) für das Lernen in Schule und Hochschule relevant gemacht werden?
}

\author{
Einleitung zur Thematik des Themenheftes
}

\author{
Julian Roelle $\mathbb{D} \cdot$ Tino Endres $(\mathbb{D} \cdot$ Alexander Renkl $(\mathbb{D}$
}

Eingegangen: 11. November 2021 / Überarbeitet: 24. November 2021 / Angenommen: 1. Dezember 2021 / Online publiziert: 17. Dezember 2021

(C) Der/die Autor(en) 2021

Zusammenfassung Es gibt eine beeindruckende Menge an Evidenz dafür, dass Abrufübungen das langfristige Behalten von Wissen bedeutend fördern können (oftmals als Testungs- oder Abrufübungseffekt bezeichnet). Es gibt allerdings auch Arbeiten, die zu kritischeren Schlüssen in Bezug auf den Nutzen von Abrufübungen kommen - insbesondere, was den Nutzen in Kontexten anbetrifft, in denen ein tiefes Verständnis komplexer Lerninhalte erworben werden soll. In diesem Beitrag werden die verschiedenen Sichtweisen auf den Nutzen von Abrufübungen mit einer zweifachen Zielsetzung reflektiert. Zum einen werden drei zentrale zu klärende Fragenkomplexe herausgearbeitet, deren Beantwortung von großer Relevanz für die breite Anwendung von Abrufübungen im Rahmen schulischen und hochschulischen Lernens wäre und es wird herausgestellt, inwiefern die Beiträge dieses Themenhefts zur Beantwortung dieser Fragenkomplexe beitragen. Zum anderen werden, ebenfalls informiert von den vier Beiträgen dieses Themenhefts, fünf Empfehlungen für Lehrkräfte formuliert, um bei der Gestaltung von Lehrveranstaltungen vom Erkenntnisstand zu Effekten von Abrufübungen profitieren zu können.

Schlüsselwörter Testungseffekt · Abrufübungen · Nachhaltiges Lernen

\footnotetext{
Julian Roelle $(\bowtie)$

Fakultät für Philosophie und Erziehungswissenschaft, Ruhr-Universität Bochum, Bochum,

Deutschland

E-Mail: julian.roelle@ruhr-uni-bochum.de

Tino Endres $\cdot$ Alexander Renkl

Abteilung für Entwicklungs- und Pädagogische Psychologie, Albert-Ludwigs-Universität Freiburg,

Freiburg, Deutschland

Tino Endres

E-Mail: tino.endres@psychologie.uni-freiburg.de

Alexander Renkl

E-Mail: renkl@psychologie.uni-freiburg.de
} 


\title{
How can retrieval practice (not) be made relevant for learning in school and higher education?
}

Introduction to the topic of the special issue

\begin{abstract}
There is an impressive body of evidence that retrieval practice can substantially enhance long-term retention of knowledge (often referred to as the testing effect or retrieval practice effect). However, there is also research that comes to more cautious conclusions concerning the benefits of retrieval practice-particularly in settings in which deep understanding of complex learning content is to be acquired. In this article, we reflect on the different perspectives on the utility of retrieval practice with a twofold objective. First, three sets of crucial open questions that are of great relevance for paving the way for the widespread use of retrieval practice in high school and higher education are identified and the extent to which the four contributions of this special issue contribute to answering these sets of questions is highlighted. Second, also informed by the four contributions of this special issue, we formulate five recommendations for teachers to benefit from the current state of knowledge concerning the effects of retrieval practice when designing courses.
\end{abstract}

Keywords Testing effect $\cdot$ Retrieval practice $\cdot$ Lasting learning

\section{Einleitung}

Das Üben des Abrufs von Informationen aus dem Langzeitgedächtnis fördert in der Regel das langfristige Behalten dieser Informationen. Dieser altgediente Testungsoder Abrufübungseffekt, der jahrzehntelang vorwiegend in der grundlagenorientierten kognitiven Psychologie untersucht wurde, hat, angestoßen durch eine vielbeachtete empirische Studie (Roediger und Karpicke 2006a) und einen vielbeachteten Übersichtsartikel (Roediger und Karpicke 2006b), in den letzten ca. 15 Jahren auch in der nutzeninspirierten pädagogisch-psychologischen Forschung ein großes Maß an Aufmerksamkeit erfahren. Diese Aufmerksamkeit ist äußerst begrüßenswert, da Abrufübungen bedeutend dazu beitragen können, dass Wissen langfristig behalten wird und im Idealfall ein Leben lang flexibel einsetzbar bleibt, was eng mit zentralen Bildungszielen in Schule und Hochschule korrespondiert. Entsprechend haben jüngere Übersichtsarbeiten und Metaanalysen unter anderem den Nutzen von Abrufübungen für das Lernen in authentischen Settings wie dem Schul- oder Hochschulunterricht in den Blick genommen (z.B. Agarwal et al. 2021; Dunlosky et al. 2013; Schwieren et al. 2017; Yang et al. 2021). Die Schlussfolgerungen dieser Arbeiten zum praktischen Nutzen von Abrufübungen für das bedeutungshaltige Lernen in Schule und Hochschule sind zumeist euphorisch. So stellen beispielsweise Dunlosky et al. (2013) Abrufübungen als eine kognitionspsychologisch begründete Lerntechnik mit hohem praktischem Nutzen heraus, was angesichts des Befunds der Metanalyse von Yang et al. (2021), in die keinerlei reine Laborstudien, sondern nur Studien, die zumindest teilweise im Klassenraum bzw. in authentischen Lernsettings durchgeführt wurden, eingeflossen sind, durchaus gerechtfertigt scheint. So finden die Autoren insgesamt einen mittelgroßen Effekt von Abrufübungen in authenti- 
schen Settings (nach Hatties 2009, Klassifizierung von Effektgrößen läge dieser Effekt deutlich über der Schwelle für wünschenswerte Effekte im Bildungsbereich), der zudem nicht nur auf den Erwerb von Faktenwissen, auf bestimmte Fächer, oder auf bestimmte Altersgruppen (siehe hierzu auch Brod 2020) begrenzt ist.

Es gibt allerdings auch Arbeiten, die zu skeptischeren Schlüssen in Bezug auf den Nutzen von Abrufübungen für bedeutungshaltiges Lernen kommen. Beispielsweise argumentieren van Gog und Sweller (2015), dass der Effekt von Abrufübungen mit zunehmender Komplexität des Lernmaterials abnimmt und für vergleichsweise komplexes Lernmaterial sogar vollständig verschwindet. Diese Argumentation basiert auf der Annahme, dass das Herstellen von Verknüpfungen zwischen zu lernenden Informationen, was ein zentraler Wirkmechanismus von Abrufübung ist, bei komplexem Material kaum nötig sei, da die Verknüpfungen im Lernmaterial bereits enthalten sind oder von den Lernenden bereits in der initialen Lernphase hergestellt würden. Angesichts dessen, dass zumindest beim fortgeschrittenen Wissenserwerb in den meisten Inhaltsdomänen komplexes Wissen erworben werden muss, würde dies den praktischen Nutzen von Abrufübungen natürlich deutlich einschränken (Carpenter et al. 2020). Darüber hinaus kritisieren van Gog und Sweller (2015; siehe auch Tran et al. 2015) die Maße, mit denen in Studien zum Testungseffekt Transfer gemessen wurde, als unzureichend, da der Transfer in Teilen sehr nah sei und zudem durch die Formulierung der Testaufgaben bedeutend unterstützt wurde. Rummer (2021) stellt zudem heraus, dass die Implementationsqualität und Passung der Lernaktivitäten, mit denen Abrufübungen in bisherigen Studien verglichen wurden, teilweise gering ist (vgl. Heitmann et al. 2021) und dass in authentischen Lernkontexten im Feld bisweilen indirekte Effekte von Abrufübungen auftreten können, die bis jetzt kaum hinreichend untersucht und verstanden worden sind.

Unter indirekten Effekten von Abrufübungen werden alle Effekte gefasst, die Abrufübungen auf das Lernen haben, das im Anschluss an die Abrufübungen stattfindet (vgl. Arnold und McDermott 2013). Dies kann sowohl das gezielte Schließen von Wissenslücken, die im Rahmen von Abrufübungen oftmals deutlich zutage treten, als auch eine geänderte Herangehensweise bei der Erarbeitung eines nächsten Themengebiets betreffen. Obgleich solche indirekten Effekte von Abrufübungen inzwischen in zahlreichen Laborstudien in den Blick genommen wurden (z. B. Arnold und McDermott 2013; Tullis et al. 2013), sind die bisherigen Befunde vermutlich nur sehr bedingt auf authentische Lernkontexte übertragbar, da sowohl das Ausmaß an leistungskontingenten Konsequenzen als auch die von den Lernenden wahrgenommene Relevanz des (intensiven) Beschäftigens mit den Inhalten zwischen Labor- und Feldkontexten in der Regel deutlich variieren dürfte (vgl. Rummer, 2021). Zudem ist es bisher insbesondere im Kontext von komplexen Abrufübungsaufgaben unklar, wie die jeweiligen anschließenden Lernphasen idealerweise gestaltet werden sollten, was der oben skizzierten Euphorie in Bezug auf die Anwendung von Abrufübungen zur Förderung des Lernens in Schule und Hochschule entgegensteht.

Die skizzierten unterschiedlichen Blickwinkel auf den Nutzen und somit die Relevanz von Abrufübungen für die Förderung des Lernens in Schule und Hochschule sind ein vielversprechender Ausgangspunkt für die Erstellung eines möglichst realistischen Bildes der Stärken und Grenzen von Abrufübungen zur Förderung bedeutungshaltigen Lernens in der pädagogischen Praxis. Im Speziellen lohnt es sich hier- 
zu, die Bedeutung der in den skeptischeren Beiträgen zum Effekt von Abrufübungen geäußerten Bedenken für die Gestaltung schulischen und hochschulischen Lernens zu reflektieren und daraus abzuleiten, welche Fragen geklärt sein müssten, damit Abrufübungen aussichtsreich angewandt werden können. Drei in diesem Kontext zentrale zu klärende Fragenkomplexe, die nicht vollständig unabhängig voneinander sind, sind aus unserer Sicht die folgenden.

\subsection{Welche spezifischen Unterrichtsziele lassen sich mit Abrufübungen erreichen?}

Selbstverständlich wird nicht einmal in den euphorischsten Beiträgen zur Nützlichkeit von Abrufübungen argumentiert, dass Abrufübungen zur Erreichung jeden Lernzieles gleich gut geeignet wären. Vergleichsweise gut empirisch belegt scheint der Nutzen von Abrufübungen für die Konsolidierung von Faktenwissen im Langzeitgedächtnis zu sein. Dies allein dürfte schon Anlass genug dafür sein, eine hohe Relevanz von Abrufübungen für die pädagogische Praxis festzustellen. Auch wenn beim Lernen in Schule und Hochschule oftmals der Erwerb von Wissen über komplexe Zusammenhänge oder Prozeduren anvisiert wird, stellt der Erwerb von Grundwissen, das für sich genommen ggf. mitunter wenig komplex ist, in der Regel einen wichtigen Schritt auf dem Weg zu diesem Ziel dar. Beispielsweise stellt der Erwerb einfacher deklarativer Konzepte in beinahe jeder Domäne einen wesentlichen Teil des zu erwerbenden Grundwissens dar, auf dem im Zuge des Erwerbs komplexeren Wissens aufgebaut wird (vgl. Rawson et al. 2015). Auch in Bezug auf Transferleistungen, die sich auf den Einsatz einfachen Faktenwissens beziehen, finden sich durchaus überzeugende förderliche Effekte von Abrufübungen in der Literatur (für eine Übersicht, siehe Pan und Rickard 2018). Zu klären ist hier allerdings, inwiefern der Transfer einfachen Faktenwissens mit auf Transfer bezogenen Unterrichtszielen korrespondiert, in denen häufig deutlich weitere Transferdistanzen in Bezug auf deutlich komplexeres Wissen anvisiert werden dürften.

Eng in Zusammenhang mit diesen offenen Punkten steht die Frage, inwiefern Abrufübungen auch für komplexes Lernmaterial nützlich sind bzw. nutzbar gemacht werden können. In diesem Kontext gilt es zum einen zu klären, wie die Komplexität von Lernmaterial so operationalisiert werden kann, dass (auch) in der Praxis fundierte Urteile zum Komplexitätsgrad und somit potenziell zur Eignung von Abrufübungen getroffen werden können. Zum anderen sollte geklärt werden, inwiefern eine potenzielle Abhängigkeit der Effektivität von Abrufübungen von der Komplexität des Lernmaterials trennbar von einer Abhängigkeit der Effekte vom Wissensstand der Lernenden zum Zeitpunkt des Einsatzes der Abrufübungen ist; für Lernende wird Lernstoff ja mit steigendem Vorwissen, zumindest in der Regel, weniger komplex (vgl. Sweller et al. 2019). Sollte im Wesentlichen der Wissensstand der Lernenden und nicht die Komplexität des Lernmaterials an sich entscheidend für die Effektivität von Abrufübungen sein, könnte es letztlich vor allem relevant sein, zu welchem Zeitpunkt im Lernprozess Abrufübungen eingesetzt werden. Im Speziellen dürften Abrufübungen in diesem Fall erst dann eingesetzt werden, wenn die Lernenden ein hinreichendes Verständnis der Lerninhalte aufbauen konnten und entsprechend eine Konsolidierung des aufgebauten Wissens sinnvoll ist. Dies würde 
aber vermutlich nicht bedeuten, dass Abrufübungen stets erst am Ende einer Unterrichtseinheit, also dann, wenn all das zu erwerbende Wissen (im Idealfall) aufgebaut wurde, eingesetzt werden sollten. Aufbauend auf dem Gedanken, dass Abrufübungen (auch) gut geeignet sind, um grundlegende Konzepte und Prinzipien, auf denen beim fortgeschrittenen Wissenserwerb aufgebaut werden soll, zu konsolidieren, wäre ein weiterer potenziell günstiger Einsatzzeitpunkt das Ende des Grundlagenteils einer Unterrichtseinheit. Ob sich diese Zeitpunkte im Vergleich zu anderen Einsatzzeitpunkten von Abrufübungen jedoch tatsächlich als förderlicher erweisen würden und ob bzw. wie sich Lehrpersonen überhaupt überzeugen ließen, auch bereits in vergleichsweise frühen Phasen ihrer Unterrichtseinheiten Abrufübungen einzusetzen, ist eine offene Frage.

Eine weitere didaktische Entscheidung, die hinsichtlich der zu erreichenden Unterrichtsziele relevant ist, betrifft das Format der Abrufübungsaufgaben. So scheinen unterschiedliche Abrufübungsaufgabenformate für unterschiedliche didaktische Ziele besonders geeignet zu sein. Aufgaben, die lediglich das Wiedererkennen von Inhalten erfordern, wie es in manchen Multiple Choice-Formaten der Fall ist, sind zumeist nur förderlich für das Wiedererkennen von Inhalten. Auf das aktive Abrufen von Wissen wirken sie sich hingegen nur selten förderlich aus (vgl. Duchastel und Nungester 1982; Veltre et al. 2015). Aber auch Abrufübungsaufgaben, die den aktiven Abruf von Wissen erfordern, dienen unterschiedlich stark verschiedenen Lernzielen. Dies betrifft sowohl die direkten wie auch indirekte Effekte (Endres et al. 2020). So fördern spezifische Abrufübungsaufgaben, die einzelne Informationen adressieren (z.B. „Was wissen Sie über den lernzielspezifischen Einsatz von Abrufübungsaufgaben?") das langfristige Behalten dieser spezifischen Inhalte und verbessern die metakognitive Überwachung. Freie Abrufübungsformate, die den Lerninhalt zusammenhängend abfragen (z. B. „Was wissen Sie über abrufbasiertes Lernen?"), scheinen hingegen das Behalten multipler Inhalte zu fördern und die Motivation der Lernenden zu steigern (vgl. Endres et al. 2020). Die Befundlage zu diesen Effekten basiert bisher allerdings nur auf einzelnen Studien, so dass es weiterer Forschung bedarf, bis belastbare Empfehlungen für die Praxis abgeleitet werden können.

\subsection{Wie kann Abrufübung mit anderen bewährten Lernaktivitäten koordiniert werden?}

Aus der Feststellung, dass Abrufübungen sich nicht zur Erreichung jedes Lernziels gleich gut eignen sollten, ergibt sich die Herausforderung, Abrufübungen mit anderen Lernaktivitäten zu koordinieren, die sich zur Erreichung von Lernzielen bewährt haben, für deren Erreichung Abrufübungen weniger gut geeignet sind. Dies kann beispielsweise die Koordinierung mit Lernaktivitäten zum Aufbau von Grundwissen in frühen Phasen des Wissenserwerbs oder zur Vertiefung des Verständnisses oder zur Automatisierung von Wissen in späteren Phasen des Wissenserwerbs betreffen. Prinzipiell ist die Koordinierung von Abrufübungen mit diesen Lernaktivitäten auf zwei Weisen denkbar. Erstens können Abrufübungen in diese Lernaktivitäten unmittelbar integriert werden, indem die jeweiligen Lernaktivitäten zumindest in Teilen ohne Zugriff auf das jeweilige Lernmaterial ausgeführt werden müssen (sog. 
Closed-Book-Format von Lernaufgaben, siehe Agarwal et al. 2008; Endres et al. 2017; Roelle und Berthold 2017) und somit simultan zu diesen Lernaktivitäten auch Abrufübungen stattfinden. Zweitens können die Lernaktivitäten sequentiell angeregt werden. Beide Arten der Koordinierung sind bisher weder aus theoretischer Sicht noch empirisch hinreichend betrachtet worden, so dass bisher keine fundierten Aussagen bzw. Empfehlungen hierzu getroffen werden können.

In Bezug auf die simultane Anregung von Abrufübungen und beispielsweise verständnisfördernden Lernaktivitäten wie dem Elaborieren von Lerninhalten stellt sich insbesondere die Frage, wie die Integration von Abrufübungen gelingen kann, ohne dass die Qualität der Ausführung der verständnisfördernden Lernaktivitäten bedeutend beeinträchtigt wird. Da der Abruf von Wissen aus dem Gedächtnis selten perfekt ist, fehlen den Lernenden bei Lernaufgaben, die im Closed-Book Format implementiert werden, oftmals einige der Informationen, die für die hochqualitative Ausführung der verständnisfördernden Lernaktivitäten zwingend benötigt werden (vgl. Hiller et al. 2020; Roelle und Berthold 2017). Beispielsweise erinnert man nicht mehr alle Details eines Konzepts, für das man sich ein eigenes Beispiel überlegen soll (Elaboration). In Bezug auf die Sequenzierung von Abrufübungen mit verständnisfördernden Lernaktivitäten stellt sich insbesondere die Frage nach der optimalen Reihenfolge. Auf den ersten Blick scheint es sinnvoll, Abrufübungen an das Ende dieser Sequenz zu setzen, um das durch die zuvor ausgeführten verständnisfördernden Lernaktivitäten vertiefte Wissen anschließend zu konsolidieren. In Abhängigkeit der Anforderungen der jeweiligen verständnisfördernden Lernaktivitäten könnte es jedoch auf den zweiten Blick ebenso sinnvoll sein, die verständnisfördernden Lernaktivitäten $\mathrm{zu}$ erleichtern, indem gewisses Grundwissen vorab konsolidiert wird. Natürlich stellt sich ebenfalls die Frage, ob die simultane oder sequentielle Anregung von Abrufübungen und weiteren Lernaktivitäten insgesamt lernförderlicher ist und von welchen Faktoren dies potenziell abhängt.

Im Kontext der Koordinierung von Abrufübungen mit verständnisfördernden Lernaktivitäten ist zudem die Frage zu klären, ob Abrufübungen hinsichtlich der Konsolidierung von Gedächtnisinhalten überhaupt einen bedeutenden Mehrwert gegenüber verständnisfördernden Lernaktivitäten hätten. Wie von Rummer (2021) dargelegt, wurden die (verständnisfördernden) Lernaktivitäten, mit denen Abrufübungen in bisherigen Studien verglichen wurden, oftmals nicht optimal implementiert bzw. nicht optimal passend zu den Lernmaterialien und Lernzielen ausgewählt. Angesichts dessen, dass aus theoretischer Sicht auch verständnisfördernde Lernaktivitäten wie das Organisieren und Elaborieren von Lerninhalten eine gewisse konsolidierende Funktion haben sollten, die auch empirisch bereits nahegelegt wurde (z. B. Berthold et al. 2007; Kintsch et al. 1990; Roelle und Nückles 2019; Waldeyer et al. 2020), sollte der Mehrwert von Abrufübungen im Vergleich zu verständnisfördernden Lernaktivitäten und Mischformen von Abrufübungen und verständnisfördernden Lernaktivitäten sorgsam geprüft werden, um die Relevanz von Abrufübungen für das Lernen in Schule und Hochschule auf ein noch solideres Fundament zu stellen. 


\subsection{Wie können indirekte Effekte von Abrufübungen im Kontext bedeutungshaltigen Lernens nutzbar gemacht werden?}

Der Effekt von Abrufübungen vergrößert sich in der Regel, wenn die Lernenden im Anschluss an die Abrufübungen eine oder mehrere weitere Lerngelegenheiten erhalten. Die dafür verantwortlichen indirekten Effekte von Abrufübungen, die durch das gezielte Nachbessern bzw. das Anpassen des Vorgehens bei der Erarbeitung nachfolgender Themengebiete entstehen, scheinen auf den ersten Blick auch in praxisnahen Studien gut realisierbar zu sein. So zeigt sich in der Metaanalyse von Yang et al. (2021) beispielsweise ein deutlicher Anstieg der Effektstärke von $g=0,37$ auf $g=0,57$, wenn mindestens eine anschließende Lerngelegenheit verfügbar war. Allerdings gibt es insbesondere dann, wenn komplexeres Wissen erworben werden soll, bisher vergleichsweise wenig empirische Evidenz in Bezug auf die optimale Gestaltung dieser Lerngelegenheiten. Ein einfaches Darstellen der korrekten Antwort bzw. Lösung, wie es in den zahlreichen Studien, in denen das Lernmaterial lediglich aus zu lernenden Wortlisten bestand, häufig praktiziert wurde, ist im Bereich des verständnisorientierten Lernens unzureichend (vgl. Pan und Rickard 2018). So können Lernende beim Bearbeiten verständnisorientierter Abrufübungsaufgaben aus multiplen Gründen fehlerhafte bzw. unvollständige Lösungen aufweisen. Eine Möglichkeit ist, dass einige Lernende eine fehlerhafte Lösung aufgrund einzelner falsch verstandener Konzepte aufweisen. Andere Lernende wiederum mögen lediglich mit der Integration der Inhalte Probleme haben, die einzelnen Inhalte aber korrekt verstanden haben. Folglich würden diese Lernenden im Grunde unterschiedliche Arten von Unterstützung zur Behebung der gleichen fehlerhaften Lösung benötigen. Eine Möglichkeit, mit dieser Herausforderung umzugehen, scheint das Bereitstellen einer größeren Bandbreite an Informationen für alle Lernenden zu sein; die Lernenden können dadurch sozusagen die Unterstützung herauspicken, die ihnen hilft, ihre individuellen Gründe für die fehlerhafte Lösung zu beheben (vgl. Endres und Renkl, dieses Themenheft).

Ein weiterer Grund, warum bisherige Laborstudienergebnisse zu indirekten Effekten von Abrufübungen für die Praxis nur in begrenztem Maße aufschlussreich sind, ist, dass sich in der Praxis die Lernmotivation aufgrund der höheren Relevanz der zu lernenden Inhalte und des Feedbacks durch die Lehrpersonen deutlich anders darstellen sollte als in Laborstudien, was die Bereitschaft, anschließende Lerngelegenheiten zu nutzen, deutlich beeinflussen dürfte (vgl. Rummer 2021; Rummer et al. 2019). Nicht nur, aber auch im Kontext der Effekte von Abrufübungen steckt die Forschung zur Regulation von Anstrengung im Kontext des (zumindest in Teilen) selbstregulierten Schließens von Wissenslücken jedoch noch in den Kinderschuhen (vgl. De Bruin et al. 2020), was ein weiteres Erschwernis in Bezug auf die Nutzbarmachung von indirekten Effekten von Abrufübungen im Kontext des Lernens in Schule und Hochschule darstellt. Auch die Rolle von Eigenschaften der Lernenden für die Effektivität erneuter Lerngelegenheiten im Anschluss an die Abrufübungen ist bisher nicht hinreichend geklärt. Beispielsweise deuten Befunde darauf hin, dass unter bestimmten Bedingungen ein hohes akademisches Selbstkonzept hinderlich, eine stark ausgeprägte Hoffnung auf Erfolg jedoch förderlich für das Ausschöpfen indirekter (und direkter) Effekte von Abrufübungen sein könnte (vgl. Heitmann et al. 
2022; Roelle und Renkl 2020), was bisher nicht eingehend betrachtet und erklärt worden ist.

Schließlich stellt sich auch die Frage, inwiefern die Gestaltung erneuter Lerngelegenheiten auch davon abhängig sein sollte, ob bei der oben skizzierten Koordinierung mit anderen, beispielsweise verständnisfördernden, Lernaktivitäten die simultane oder sequentielle Variante realisiert wird. Im Fall einer simultanen Realisierung müssten beispielsweise gegebenenfalls Wege gefunden werden, die erneute Lerngelegenheit so zu gestalten, dass sie nicht nur für die Abrufübungen, sondern auch für die jeweils andere ausgeführte Lernaktivität eine hilfreiche weitere Lerngelegenheit darstellt. Im Falle einer sequentiellen Realisierung könnte eine Auseinandersetzung damit sinnvoll sein, ob den Lernenden jeweils kommuniziert werden sollte, für welche jeweils nächste Lernaktivität sie die Abrufübungen und die erneute Lerngelegenheit vorbereiten sollen. Dies könnte insbesondere die Art, wie Lernende die erneute Lerngelegenheit nutzen, förderlich beeinflussen (im Sinne einer generellen Relevanzinstruktion, vgl. McCrudden und Schraw 2007).

\section{Beiträge des Thementeils zu den genannten Fragenkomplexen}

Der Thementeil umfasst vier Arbeiten, in denen die drei skizzierten Fragenkomplexe behandelt werden. Barenberg und Dutke (dieses Themenheft) beziehen sich insbesondere auf den ersten Fragenkomplex und argumentieren, dass der Testungseffekt insgesamt gesehen ein sehr gut etablierter Befund ist. Insofern sollten - entsprechend der Ansätze, die Lehre gemäß der besten verfügbaren Forschungsevidenz zu gestalten suchen (evidence-based teaching) - Testaufgaben nicht nur zu Zwecken der Diagnostik, sondern auch zu Zwecken der Lernförderung eingesetzt werden. Zumindest aber ist die Strategie, Testaufgaben zur Lernförderung einzusetzen, ein wissenschaftlich begründetes Angebot an die Praxis. Die Autoren führen drei zentrale Argumente dafür auf, warum Testen zur Lernförderung im Unterricht seinen Platz finden sollte, wobei ihre Betrachtung in erster Linie die direkten Testungseffekte fokussiert (zu indirekten Effekten siehe Endres und Renkl, dieses Themenheft). Erstens wurden die zunächst eher grundlagenorientierten Studien zu Testungseffekten durch zahlreiche Untersuchungen in anwendungsnahen Lehr-Lernkontexten ergänzt, die ebenfalls positive Wirkungen des Testens zeigen. Zweitens ergeben sich aus der Vorgabe von Testaufgaben zum Teil auch Transfereffekte; so werden beispielsweise bei kohärentem Lernmaterial, wie es für Schule und Hochschule typisch ist (zusammenhängende Darstellung eines Sachverhalts in Vergleich zum Erlernen einer Liste einzelner Vokabeln), nicht nur die abgetesteten Informationen besser behalten, sondern auch damit in Zusammenhang stehende Informationen. Drittens, wird die eigene Einschätzung des aktuellen Wissensstands (metakognitives Monitoring), die sehr oft positiv verzerrt ist, durch die Vorgaben von Tests realistischer. Da eine realistische metakognitive Einschätzung insbesondere für weitere Lernprozesse, in denen z. B. identifizierte Wissenslücken geschlossen werden können, wichtig ist, steht dieser Effekt eng mit indirekten Testungseffekten in Bezug.

Rummer und Schweppe (dieses Themenheft) befassen sich ebenfalls vorwiegend mit dem ersten Fragenkomplex. Im Speziellen setzen sie sich eingehend mit der 
Frage auseinander, ob der Nutzen von Abrufübungen von der Komplexität der zu lernenden Inhalte abhängig ist. Die Autor*innen problematisieren dabei den Komplexitätsbegriff, der in vorheriger Forschung verwendet worden ist und argumentieren, dass zunächst zwischen der Komplexität des Lernstoffs und der Komplexität der instruktionalen Umsetzung des Lernstoffs unterschieden werden müsse. Zudem stellen sie heraus, dass in keinem der Experimente, auf die sich van Gog und Sweller (2015) bei ihrer Argumentation beziehen, dass Abrufübungen bei komplexen Lerninhalten kaum einen Nutzen haben, tatsächlich die Komplexität des Lernstoffs manipuliert wurde. Darüber hinaus unterbreiten Rummer und Schweppe eine umfassende Alternativerklärung für den Einfluss von Komplexität (des Lernstoffs und des Lernmaterials) auf die Effektivität von Abrufübungen. So argumentieren die Autor*innen, dass komplexe Inhalte schwerer verständlich seien und Lernende entsprechend eine längere initiale Lernphase vor den Abrufübungen benötigen würden, um ein hinreichendes Verständnis aufzubauen, dessen Konsolidierung durch Abrufübungen sich lohnen würde. Folglich könnten bei komplexen Lerninhalten die Abrufübungen in bisherigen Studien jeweils zu früh angesetzt gewesen sein, wodurch der Abruf unvollständig und fehlerhaft gewesen sein mag, was sich abträglich auf den Lernerfolg ausgewirkt haben könnte. Abrufübungen sind schließlich vor allem dann lernförderlich, wenn den Lernenden der Abruf zu weiten Teilen gelingt (vgl. Karpicke 2017). Rummer und Schweppe skizzieren darüber hinaus, wie diese Annahme experimentell überprüft werden könnte und warum für den Zeitpunkt von Abrufübungen im Lernprozess eine Balance zwischen „so früh wie möglich“ und „nicht zu früh“ gefunden werden müsse.

Der Beitrag von Roelle und Nückles (dieses Themenheft) verhandelt im Wesentlichen die Frage, wie Abrufübungen mit bewährten Wissenskonstruktionsaktivitäten wie dem Organisieren, Elaborieren und Generieren koordiniert werden können (Fragenkomplex 2). Die Autoren stellen heraus, dass positive Effekte der simultanen Anregung von Abrufübungen und Wissenskonstruktionsaktivitäten durch ein ClosedBook-Format von auf Konstruktion zielenden Lernaufgaben von einer zentralen Bedingung abhängig sind. Es sollten flankierende Maßnahmen getroffen werden, um abträgliche Effekte von nicht abrufbaren oder falsch abgerufenen Informationen auf die Qualität der Wissenskonstruktionsaktivitäten zu verhindern. Vielversprechende Maßnahmen hierzu seien unter anderem eine Vertiefung bzw. Fokussierung der Verarbeitung vor den Abrufübungen oder das Einbauen von Möglichkeiten, das Lernmaterial während oder nach den Abrufübungen erneut einzusehen. Die Autoren sehen den Bedarf für solche Maßnahmen insbesondere bei der Koordinierung von Abrufübungen mit vergleichsweise komplexen auf Konstruktion zielenden Lernaktivitäten, wobei sie auch aufzeigen, dass die abträglichen Folgen eines ClosedBook-Formats von auf Konstruktion zielenden Lernaufgaben bisher nur für einige wenige Typen von auf Konstruktion zielenden Lernaufgaben eingehend untersucht worden sind. Darüber hinaus kritisieren Roelle und Nückles den dürftigen Theoriebezug bisheriger Forschung, die sich mit der Integration von Abrufübungen in auf Konstruktion zielende Lernaufgaben beschäftigt und argumentieren, dass neben der bisher vorwiegend in den Blick genommenen simultanen Anregung von Abrufübungen und Wissenskonstruktionsaktivitäten auch eine Sequenzierung der beiden Arten von Lernaktivitäten vielversprechend sein könnte. 
Endres und Renkl (dieses Themenheft) beschäftigen sich schließlich mit dem dritten Fragenkomplex und wollen dazu beitragen, indirekte Effekte von Abrufübungen im Kontext des Lernens in Schule und Hochschule besser nutzbar zu machen. Dabei greifen die Autoren verschiedene mögliche Lernziele auf und erläutern, wie diese Effekte durch eine geeignete Abrufübungsaufgabenauswahl und -implementierung in der Praxis genutzt werden können. Sie stellen heraus, dass Abrufübungsaufgaben die metakognitive Genauigkeit sowie die Nutzung der metakognitiven Informationen verbessern können und dass Abrufübungsaufgaben Einfluss auf motivationale Komponenten haben bzw. Lernende motivieren können, engagiert erneut zu lernen. Zuletzt stellen sie heraus, dass auch kognitive Komponenten der Lernzielkommunikation und der Aktivierung des Vorwissens relevant für die indirekten Effekte von Abrufübungen sind. In der Zusammenstellung wird deutlich, dass es nicht eine ideale Art gibt, indirekte Effekte von Abrufübungen zu nutzen. Je nach Lernzielen und den Eigenschaften der Lernenden sind unterschiedliche Arten der Nutzung indirekter Effekte von Abrufübungen zu empfehlen, da unterschiedliche Abrufarten jeweils einzigartige Stärken und Herausforderungen in Bezug auf bestimmte Ziele mit sich bringen. Um die jeweiligen Vorteile ideal zu nutzen, sollten sowohl Lehrende wie auch Lernende um die Wirkmechanismen der indirekten Effekte der Abrufübungen wissen. Entsprechend erläutern Endres und Renkl eingehend die angenommenen Wirkmechanismen, um informierte Entscheidungen zu ermöglichen, wie das eigene Lernverhalten oder die Implementierung durch Lehrende gezielt optimiert werden kann.

\section{Und was bleibt: Ein Denkanstoß für die Praxis oder eine klare Empfehlung?}

Die Beiträge zum Testungseffekt in diesem Themenheft sind sich einig, dass die Forschung klar gezeigt hat, dass Testen in direkter und indirekter Weise das Lernen fördern kann. Sie nehmen aber eine etwas unterschiedliche Position dazu ein, unter welchen Umständen man den Einsatz von Abrufübungen empfehlen sollte. Auch wenn Barenberg und Dutke (dieses Themenheft) anerkennen, dass Wissenschaft nur Angebote für die Praxis machen kann, so geht doch der Tenor des Beitrags hin zu einer Empfehlung, Abrufübungen breit im Unterricht einzusetzen. Rummer und Schweppe (dieses Themenheft) sowie Roelle und Nückles (dieses Themenheft) sind etwas vorsichtiger und fordern in Hinblick auf die Anwendung weitere Forschung ein, etwa zu den Effekten des Testens bei unterschiedlich komplexem Lernmaterial oder bei der Kombination des Testens mit anderen verständnisfördernden Lernaktivitäten im Unterricht. Endres und Renkl (dieses Themenheft) betonen vor allem die Aspekte, dass Lehrkräfte in Hinblick auf Merkmale ihrer Lernenden und auf ihre Hauptziele des Unterrichts entscheiden müssen, ob und vor allem wie sie das Testen zur Lernförderung einsetzen; es geht sozusagen um eine interpretative Nutzung der Befunde.

Einig sind sich die Autor*innen, dass der Befund, dass Testen Lernen fördert, von Lehrkräften sinnvollerweise berücksichtigt werden sollte. Es sind jedoch einige Fragen offen, wann es darum geht, wie man das Testen zur Lernförderung im Unterricht 
genau einsetzen sollte. Dies gilt insbesondere, wenn man wichtige Entscheidungen, die die Lehrkraft zu treffen hat, mitberücksichtigt, etwa über die Art der priorisierten Lernziele, über den didaktischen Ort des Testens, über die Kombination oder Sequenzierung mit anderen lernförderlichen Aktivitäten, über die Berücksichtigung individueller Lernvoraussetzungen oder über die genaue Art der Testaufgaben. Vor dem Hintergrund dieser komplexen Entscheidungssituation wäre es zweifelsfrei von großem Nutzen, weitere Befunde zu entscheidungsrelevanten Faktoren beim Einsatz des Testens zur Lernförderung zu haben, wie sie in allen Beiträgen dieses Themenhefts, wenn auch in unterschiedlichem Ausmaß, eingefordert wurden.

Es sollte jedoch klar sein, dass es eine Illusion sein dürfte, dass die Forschung für sehr komplexe Entscheidungssituationen, wie sie Unterrichtsplanung und -gestaltung darstellen, so robuste, detaillierte und ,vollständige“ Befundmuster liefern kann, dass eine Lehrkraft nicht weitere Ressourcen, wie etwa ihr Erfahrungswissen und ihr Wissen über im gegebenen Kontext zu erreichende Zielkonfigurationen, für Entscheidungen mit einbeziehen müsste (z.B. Biesta 2007; Stark 2017; vgl. auch Cronbrach's 1975, bekanntes Problem des hall of mirrors oder die gescheiterte Idee eines teacher-proof currciculum, z. B. Priestley et al. 2015). An dieser Stelle sollte ebenfalls bemerkt werden, dass auch das Konzept der evidenzbasierten Medizin - welches im Bildungsbereich vielfach als Modell angesehen wird - nicht annimmt, dass die Wissenschaft vollständige Antworten für die Praxis gibt. Die vielzitierte Arbeit von Sackett et al. (1996) mit dem Titel „Evidence based medicine: what it is and what it isn't“ beginnt mit den Satz „It's about integrating individual clinical expertise and the best external evidence."

Insofern ist es nicht sinnvoll, den Rückgriff auf das bereits jetzt relativ reichhaltige Wissen über Testungseffekte bei der Planung und Durchführung von Unterrichts auf den Sankt Nimmerleinstag zu vertagen, an dem vermeintlich alles geklärt sein wird. An dieser Stelle können keine konkreten Richtlinien für die Nutzung des Testungseffekts im Unterrichts ausgearbeitet werden. Wir formulieren aber fünf generelle Empfehlungen an Lehrkräfte, um vom Erkenntnisstand zu Testungseffekten zu profitieren.

1. Die naheliegendste Empfehlung ist es, neben der Förderung des Verstehens die Konsolidierung des Wissens mittels Testaufgaben oder Abrufanforderungen zu fördern, da Lernende möglichst nachhaltiges Wissen erwerben sollten. Lernaktivitäten zur Verständnisförderung führen nicht automatisch zum nachhaltigen Wissenserwerb. Insofern ist ein Ausnutzen des Testungseffekts im Unterricht als wichtige Ergänzung zur Verständnisförderung sinnvoll.

2. Abrufübungen sollten, speziell bei komplexem und schwierigem Lernstoff nicht zu früh eingesetzt werden. Dies hat insbesondere zwei Gründe. Zum einen haben Abrufübungen die besten Effekte, wenn die Mehrzahl der Testaufgaben korrekt beantwortet werden kann. Zum anderen ist es sinnvoll, nicht rudimentäres und ggf. noch partiell fehlerhaftes Wissen zu konsolidieren, sondern vernetzte und Verständnis konstituierende Wissensstrukturen.

3. Die Unterrichtszeit in einem Fach ist eine begrenzte Ressource. Insofern können Lehrkräfte in Planungskonflikte kommen, wenn sie die Empfehlung, (zusätzliche) Abrufübungen im Unterricht zu intergieren, umsetzten wollen. Sie mögen über- 
legen, was sie weglassen könnten, wenn sie zusätzliche Testaufgaben einsetzten oder closed book-Lernaktivitäten nutzen wollen, die ggf. zu mehr Schwierigkeiten auf Seiten der Lernenden und damit Zeitbedarf führen. Hier könnte fachdidaktisches Wissen helfen, vor allem dazu, welche Stoffaspekte in späteren Unterrichtseinheiten wieder als wichtige Lernvoraussetzung gebraucht werden (z. B. physikalisches Wissen über lineare Bewegungen in späteren Einheiten über Kreisbewegungen). Insofern kann bei Zeitkonflikten vor allem der später nochmals besonders wichtige Lernstoff fokussiert werden, wenn Tests und Abrufanforderungen zusätzlich in den Unterricht integriert werden sollen.

4. Lehrkräfte sollten das Wissen über Testungseffekte nicht nur berücksichtigen, wenn sie typische Lernaufgaben aus der Testungseffektforschung einsetzen (wollen), sie sollten sich vielmehr generell der positiven, aber ggf. auch negativen Effekte eines (notwendigen) Gedächtnisabrufes bewusst sein, auch wenn sie ,alt hergebrachte" Unterrichtsaktivitäten (z.B. Aktivierung von Wissen über bereits behandelten Stoff in Unterrichtsgespräch, Bearbeiten von Übungsaufgaben in Stillarbeit) einsetzen oder Lernaktivitäten integrieren, die in den letzten Jahrzehnten teils im Unterricht ihren Platz fanden (z. B. Concept Mapping). Je nach konkreter Gestaltung (z. B. bei einem closed book-Format) wird substantieller Gedächtnisabruf gefordert. Dieser Gedächtnisabruf kann wünschenswerte Lerneffekte bewirken. Insofern kann es sinnvoll sein, ein closed book-Format zu wählen, wenn eine Concept Map erstellt werden soll. Zugleich sollte sich die Lehrkraft aber bewusst sein, dass dieses Format den Nachteil haben kann, dass die Lernenden relevante Information nicht erinnern, wenn der entsprechende Stoff noch nicht gut genug „,sitzt“. Im Falle des Concept Mapping können natürlich keine hochwertigen Maps entstehen, wenn wichtige Konzept nicht mehr erinnert werden. Mit anderen Worten, die Literatur zum Testungseffekt gibt Lehrkräften Wissen an die Hand, das ihnen „besser informierte“ Entscheidungen darüber treffen lässt, wie sie in einer Unterrichtseinheit z. B. die Aufgabenbearbeitung in Stillarbeit oder eine Concept Mapping-Aktivität gestalten sollten.

5. Wie die Forschung zu indirekten Effekten zeigt, spielen bei der Nutzung des Testungseffekts nicht nur die kognitiven Lerneffekte im engeren Sinne eine Rolle. So kann man zwar mit spezifischen Testfragen besonders wichtigen Stoff (z.B. für spätere Unterrichtseinheiten) gezielt konsolidieren. Dies kann aber ungünstige motivationale Auswirkungen etwa auf das Interesse bei den Lernenden haben. Unspezifische Testfragen vermeiden dies, haben aber wieder andere Nachteile (z.B. wichtige Stoffaspekte mögen nicht abgerufen werden). Die Forschung zum Testungseffekt zeigt Lehrkräften also potenzielle Nachteile bestimmter Entscheidungen auf und sensibilisiert sie für mögliche Probleme, die im Unterricht auftreten können. Sie können dann im Unterricht darauf achten, wie stark die potenziellen Nachteile im ihrem Kontext auftreten und ggf. gezielte Gegenmaßnehmen treffen.

Zusammengefasst kann man festhalten, dass die Forschung zum Testungseffekt reichhaltiges praxisrelevantes Wissen bereitstellt. Dennoch können daraus keine spezifischen Handlungsempfehlungen mit genereller Gültigkeit für Lehrkräfte abgeleitet werden. Lehrkräfte können das Wissen aber nutzen, um informierte Entscheidungen zu treffen, etwa wenn sie Wissen konsolidieren wollen oder andere Lernaktivitäten 
einsetzen, die ggf. mit Abrufanforderungen kombiniert werden können. Da dieser Forschungszweig sehr aktiv ist, wird das Wissen für informierte Entscheidungen in den nächsten Jahren substantiell zunehmen. Eine substantielle Herausforderung für die Zukunft ist es, wie man das komplexe Wissen über die Effekte von Abrufübungen Lehrkräften so vermitteln kann, dass sie dieses für ihre Praxis nutzbar machen können. Das vorliegende Themenheft dient (hoffentlich) als ein erster Schritt in dieser Richtung.

Funding Open Access funding enabled and organized by Projekt DEAL.

Open Access Dieser Artikel wird unter der Creative Commons Namensnennung 4.0 International Lizenz veröffentlicht, welche die Nutzung, Vervielfältigung, Bearbeitung, Verbreitung und Wiedergabe in jeglichem Medium und Format erlaubt, sofern Sie den/die ursprünglichen Autor(en) und die Quelle ordnungsgemäß nennen, einen Link zur Creative Commons Lizenz beifügen und angeben, ob Änderungen vorgenommen wurden.

Die in diesem Artikel enthaltenen Bilder und sonstiges Drittmaterial unterliegen ebenfalls der genannten Creative Commons Lizenz, sofern sich aus der Abbildungslegende nichts anderes ergibt. Sofern das betreffende Material nicht unter der genannten Creative Commons Lizenz steht und die betreffende Handlung nicht nach gesetzlichen Vorschriften erlaubt ist, ist für die oben aufgeführten Weiterverwendungen des Materials die Einwilligung des jeweiligen Rechteinhabers einzuholen.

Weitere Details zur Lizenz entnehmen Sie bitte der Lizenzinformation auf http://creativecommons.org/ licenses/by/4.0/deed.de.

\section{Literatur}

Agarwal, P. K., Karpicke, J. D., Kang, S.H. K., Roediger, H. L., \& McDermott, K. B. (2008). Examining the testing effect with open- and closed-book tests. Applied Cognitive Psychology, 22, 861-876. https:// doi.org/10.1002/acp.1391.

Agarwal, P. K., Nunes, L. D., \& Blunt, J.R. (2021). Retrieval practice consistently benefits student learning: a systematic review of applied research in schools and classrooms. Educational Psychology Review. https://doi.org/10.1007/s10648-021-09595-9. Advance online publication.

Arnold, K. M., \& McDermott, K. B. (2013). Test-potentiated learning: distinguishing between direct and indirect effects of tests. Journal of Experimental Psychology: Learning, Memory, and Cognition, 39(3), 940-945. https://doi.org/10.1037/a0029199.

Berthold, K., Nückles, M., \& Renkl, A. (2007). Do learning protocols support learning strategies and outcomes? The role of cognitive and metacognitive prompts. Learning and Instruction, 17, 564-577. https://doi.org/10.1016/j.learninstruc.2007.09.007.

Biesta, G. (2007). Why "what works" won't work: evidence-based practice and the democratic deficit in educational research. Educational theory, 57(1), 1-22. https://doi.org/10.1111/j.1741-5446.2006. 00241.x.

Brod, G. (2020). Generative learning: which strategies for what age? Educational Psychology Review. https://doi.org/10.1007/s10648-020-09571-9.

Carpenter, S. K., Endres, T., \& Hui, L. (2020). Students' use of retrieval in self-regulated learning: implications for monitoring and regulating effortful learning experiences. Educational Psychology Review, 32(4), 1029-1054.

Cronbach, L. J. (1975). Beyond the two disciplines of scientific psychology. American Psychologist, 30(2), 116-127. https://doi.org/10.1037/h0076829.

De Bruin, A. B. H., Roelle, J., Carpenter, S., Baars, M., \& EFG-MRE (2020). Synthesizing cognitive load and self-regulation theory: a theoretical framework and research agenda. Educational Psychology Review, 32, 903-915. https://doi.org/10.1007/s10648-020-09576-4.

Duchastel, P., \& Nungester, R. (1982). Testing effects measured with alternate test forms. The Journal of Educational Research, 74(1), 18. https://doi.org/10.1080/00220671.1982.10885400. 
Dunlosky, J., Rawson, K. A., Marsh, E. J., Nathan, M., \& Willingham, D. T. (2013). Improving students' learning with effective learning techniques: promising directions from cognitive and educational psychology. Psychological Science in the Public Interest, 14, 4-58. https://doi.org/10.1177/ 1529100612453266.

Endres, T., Carpenter, S., Martin, A., \& Renkl, A. (2017). Enhancing learning by retrieval: enriching free recall with elaborative prompting. Learning and Instruction, 49, 13-20. https://doi.org/10.1016/j. learninstruc.2016.11.010.

Endres, T., Kranzdorf, L., Schneider, V., \& Renkl, A. (2020). It matters how to recall-task differences in retrieval practice. Instructional Science, 48(6), 699-728.

Hattie, J. (2009). Visible learning: a synthesis of over 800 meta-analyses relating to achievement. London: Routledge.

Heitmann, S., Grund, A., Fries, S., Berthold, K., \& Roelle, J. (2022). The quizzing effect depends on hope of success and can be optimized by cognitive load-based adaptation. Learning and Instruction, 77, 101526. https://doi.org/10.1016/j.learninstruc.2021.101526.

Heitmann, S., Obergassel, N., Fries, S., Grund, A., Berthold, K., \& Roelle, J. (2021). Adaptive practice quizzing in a university lecture: a pre-registered field experiment. Journal of Applied Research in Memory and Cognition. https://doi.org/10.1016/j.jarmac.2021.07.008.

Hiller, S., Rumann, S., Berthold, K., \& Roelle, J. (2020). Example-based learning: should learners receive closed-book or open-book self-explanation prompts? Instructional Science, 48, 623-649. https://doi. org/10.1007/s11251-020-09523-4.

Karpicke, J. D. (2017). Retrieval-based learning: a decade of progress. In J.H. Byrne (Hrsg.), Learning and memory: a comprehensive reference (2. Aufl. S. 487-514). New York, Boston, London, Oxford: Academic Press. https://doi.org/10.1016/B978-0-12-809324-5.21055-9.

Kintsch, W., Welsch, D., Schmalhofer, F., \& Zimny, S. (1990). Sentence memory: a theoretical analysis. Journal of Memory and Language, 29, 133-159. https://doi.org/10.1016/0749-596X(90)90069-C.

McCrudden, M. T., \& Schraw, G. (2007). Relevance and goal-focusing in text processing. Educational Psychology Review, 19, 113-139. https://doi.org/10.1007/s10648-006-9010-7.

Pan, S. C., \& Rickard, T. C. (2018). Transfer of test-enhanced learning: meta-analytic review and synthesis. Psychological Bulletin, 144(7), 710-756. https://doi.org/10.1037/bul0000151.

Priestley, M., Biesta, G. J. J., Philippou, S., \& Robinson, S. (2015). The teacher and the curriculum: exploring teacher agency. In D. Wyse, L. Hayward \& J. Pandya (Hrsg.), The SAGE handbook of curriculum, pedagogy and assessment (S. 187-201). London: SAGE. https://doi.org/10.4135/9781473921405. n12.

Rawson, K. A., Thomas, R.C., \& Jacboy, L.L. (2015). The power of examples: illustrative examples enhance conceptual learning of declarative concepts. Educational Psychology Review, 27, 483-504. https://doi.org/10.1007/s10648-014-9273-3.

Roediger III, H. L., \& Karpicke, J. D. (2006a). Test-enhanced learning: taking memory tests improves longterm retention. Psychological Science, 17(3), 249-255. https://doi.org/10.1111/j.1467-9280.2006. 01693.x.

Roediger, H.L., \& Karpicke, J. D. (2006b). The power of testing memory: basic research and implications for educational practice. Perspectives on Psychological Science, 1, 181-210. https://doi.org/10.1111/ j.1745-6916.2006.00012.x.

Roelle, J., \& Berthold, K. (2017). Effects of incorporating retrieval into learning tasks: the complexity of the tasks matters. Learning and Instruction, 49, 142-156. https://doi.org/10.1016/j.learninstruc.2017. 01.008 .

Roelle, J., \& Nückles, M. (2019). Generative learning versus retrieval practice in learning from text: the cohesion and elaboration of the text matters. Journal of Educational Psychology, 111, 1341-1361. https://doi.org/10.1037/edu0000345.

Roelle, J., \& Renkl, A. (2020). Does an option to review instructional explanations enhance examplebased learning? It depends on learners' academic self-concept. Journal of Educational Psychology, 112, 131-147. https://doi.org/10.1037/edu0000365.

Rummer, R. (2021). Der Testungseffekt beim Lernen mit Texten: Ein Beispiel für das schwierige Verhältnis zwischen Grundlagenforschung und Anwendung. Psychologische Rundschau, 72, 259-272. https:// doi.org/10.1026/0033-3042/a000518.

Rummer, R., Schweppe, J., \& Schwede, A. (2019). Open-book versus closed-book tests in university classes: a field experiment. Frontiers in Psychology, 10, 463. https://doi.org/10.3389/fpsyg.2019.00463.

Sackett, D. L., Rosenberg, W. M., Gray, J. M., Haynes, R. B., \& Richardson, W. S. (1996). Evidence based medicine: what it is and what it isn't. British Medical Journal, 312(7023), 71-72. https://doi.org/10. 1136/bmj.312.7023.71. 
Schwieren, J., Barenberg, J., \& Dutke, S. (2017). The testing effect in the psychology classroom: a metaanalytic perspective. Psychology Learning and Teaching, 16(2), 179-196. https://doi.org/10.1177/ 1475725717695149.

Stark, R. (2017). Probleme evidenzbasierter bzw.-orientierter pädagogischer Praxis. Zeitschrift für Pädagogische Psychologie, 31, 99-110. https://doi.org/10.1024/1010-0652/a000201.

Sweller, J., van Merriënboer, J.J.G., \& Paas, F. (2019). Cognitive architecture and instructional design: 20 years later. Educational Psychology Review, 31(2), 261-292. https://doi.org/10.1007/s10648-01909465-5.

Tran, R., Rohrer, D., \& Pashler, H. (2015). Retrieval practice: the lack of transfer to deductive inferences. Psychonomic Bulletin and Review, 22, 135-140. https://doi.org/10.3758/s13423-014-0646-X.

Tullis, J.G., Finley, J.R., \& Benjamin, A.S. (2013). Metacognition of the testing effect: guiding learners to predict the benefits of retrieval. Memory and Cognition, 41(3), 429-442. https://doi.org/10.3758/ s13421-012-0274-5.

Van Gog, T., \& Sweller, J. (2015). Not new, but nearly forgotten: the testing effect decreases or even disappears as the complexity of learning materials increases. Educational Psychology Review, 27, 247-264. https://doi.org/10.1007/s 10648-015-9310-x.

Veltre, M. T., Cho, K. W., \& Neely, J.H. (2015). Transfer-appropriate processing in the testing effect. $M e$ mory, 23(8), 1229-1237. https://doi.org/10.1080/09658211.2014.970196.

Waldeyer, J., Heitmann, S., Moning, J., \& Roelle, J. (2020). Can generative learning tasks be optimized by incorporation of retrieval practice? Journal of Applied Research in Memory and Cognition, 9, 355-369. https://doi.org/10.1016/j.jarmac.2020.05.001.

Yang, C., Luo, L., Vadillo, M. A., Yu, R., \& Shanks, D.R. (2021). Testing (quizzing) boosts classroom learning: a systematic and meta-analytic review. Psychological Bulletin. https://doi.org/10.1037/ bul0000309. Advance online publication. 